\title{
Aceite de maracuyá (Passiflora edulis): Aprovechamiento de las semillas en productos cosméticos
}

\author{
(Passion fruit oil (Passiflora edulis): \\ Use of the seeds in cosmetic products)
}

\author{
Janeth Proaño, ${ }^{1}$ Erika Rivadeneira, ${ }^{2}$ Pablo Moncayo, ${ }^{3}$ Elizabeth Mosquera ${ }^{4}$
}

\section{Resumen}

El trabajo enfoca el aprovechamiento de las semillas de maracuyá Passiflora edulis f. flavicarpa Deg. con aplicación en el diseño de un producto cosmético: crema exfoliante con aceite de semillas de maracuyá y residuo sólido generado de la extracción del aceite. La metodología aplicada para la formulación de la crema consistió inicialmente en la extracción del aceite de las semillas por solventes orgánicos, éter etílico y por metodología mecánica a través del prensado. Las variables físico-químicas de análisis evaluadas en la crema exfoliante fueron el pH, la extensibilidad y la capacidad de emulsión; entre las propiedades físicas se evaluó el color, olor, brillo, arenosidad y ausencia de grumos como indicadores macroscópicos de una adecuada distribución del tamaño de la partícula y de una textura uniforme. Se concluyó que el aceite de las semillas de maracuyá, por su composición en elementos proteicos y lipídicos, conservan de manera óptima las propiedades inherentes a un producto diseñado para el cuidado y acondicionamiento de la piel. Esta alternativa de uso, proveniente de material que para las industrias primarias es un residuo no aprovechable, representa una alternativa en la gama de materias primas semielaboradas con propiedades que agregan valor a productos para el consumo humano.

\section{Palabras clave}

Maracuyá; aceite de semilla; crema exfoliante; cosmético; residuo agroindustria.

\begin{abstract}
The work documented in this essay is focused on harnessing the properties of Passion fruit seeds Passiflora edulis f.flavicarpa Deg to achieve the design of a cosmetic product: an exfoliating cream containing passion fruit seed oil and solid residue generated from oil extraction. The applied methodology for the formulation of the cream was initially based on a combination of a mechanical process through pressing, and oil extraction from the seeds by the use of organic solvents, ethyl ether. The physicochemical analysis variables evaluated in the exfoliating cream were $\mathrm{pH}$, extensibility and emulsion capacity. The physical properties were considered as listed, colour, odour, brightness and grittiness. Furthermore, the absence of lumps was evaluated as macroscopic indicators of adequate particle size distribution and uniform texture. It was concluded that the oil from passionfruit seeds due to its composition in protein and liquid elements, optimally conserves the inherent properties of a product designed for skincare and conditioning. The use of solid residue that for primary industries is considered as unstable waste, represents an innovative alternative in the range of available semi-finished raw materials with properties capable of enhancing the products for human consumption.
\end{abstract}

\section{Keywords}

Passion fruit; seed oil; exfoliating cream; cosmetic; agroindustrial waste.

\section{Introducción}

La investigación enfoca el aprovechamiento de las semillas de maracuyá con aplicación en el campo de la cosmetología. El objetivo del trabajo fue desarrollar un producto, crema exfoliante 
con aceite de semillas de maracuyá y el residuo sólido generado de la extracción del aceite. Passiflora edulis f flavicarpa Deg., conocida como la fruta de la pasión, tiene su origen en Sudamérica y es considerada una fruta exótica de suma importancia en el mundo dada su composición bioquímica (García, 2002).

La transformación industrial de maracuyá genera una considerable cantidad de residuo sólido que contiene a la semilla, este subproducto agrícola es utilizado para la elaboración de abonos orgánicos, piensos para animales o enviados a rellenos sanitarios (Pantoja, Hurtado y Martínez, 2017). De la totalidad de fruta procesada, se estima que la fracción residual alcanza entre 61-86 \% (Malacrida y Neuza, 2012). Estos residuos pueden ser aprovechados para la obtención de productos de interés industrial al generar características que les agregan valor y contribuyen a mitigar el impacto sobre el ambiente que generan por un manejo inadecuado. Entre los productos de interés, que en la actualidad son fabricados a partir de maracuyá figuran las pectinas, los aceites vegetales y los aromas naturales (Leao, Sampayo, Pagani y Da Silva, 2014).

Varios estudios concluyen que las semillas de maracuyá contienen una apreciable cantidad de aceite misma que varía entre 16.7-33.5 \% en función de la metodología de extracción, el tipo de solvente utilizado, las condiciones de operación y la zona geográfica de cultivo de la fruta (Malacrida y Neuza, 2012; Oliveira, Angonesea, Gomez y Ferreira, 2016; Cerón, Osorio y Hurtado, 2012).

El perfil lipídico del aceite presente en las semillas de maracuyá lo cataloga como de alto valor nutricional dada la composición de los elementos grasos como los ácidos linoleico, oleico y palmítico (Liu, Xu, Gong, He y Gao, 2012). El aceite muestra un perfil de ácidos grasos predominantemente insaturados y sobresale el linoleico, ácido graso esencial para el organismo humano (Da Silva y Neuza, 2014). Se destaca además, la característica antioxidante descrita en los estudios de (Malacrida y Neuza, 2012) y, es considerado una fuente promisoria en aplicaciones dentro del campo de la industria alimentaria, farmacéutica y cosmética (Zeraik, Pereira, Zuin y Yariwake, 2010; Malacrida y Neuza, 2012).

La similitud de la composición del aceite de semillas de maracuyá con aceites de uso comestible o de uso en la industria cosmética, hacen de este aceite una alternativa para el diseño de productos como cremas exfoliantes (Pantoja, Hurtado y Martínez, 2017). El aceite y los residuos sólidos de las semillas de maracuyá agregados a una crema base en diferentes proporciones, no afectan a las propiedades físico-químicas de las cremas cosméticas.

La fabricación de crema exfoliante con componentes de interés, comporta una serie de parámetros a cumplir como la estabilidad física del sistema, la estabilidad química del principio activo, el pH, la viscosidad, la difusión del principio activo, entre otros aspectos (Lillini, Pasquali, Pedemonte, Bregni y Lavaselli, 2016). Las cremas forman parte del grupo de productos de mayor uso y es el más común entre las lociones, emulsiones y pomadas; las que deben poseer condiciones definidas por el pH, la extensibilidad, capacidad de emulsión, entre otros, para su aplicación en la piel (Lillini, Pasquali, Pedeminte, Bregni y Lavaselli, 2016).

El aceite de maracuyá es una fuente alternativa de aceites vegetales obtenido de los residuos generados del procesamiento de la fruta, el cual puede servir como material bruto para la industria alimentaria, química y farmacéutica (Da Silva y Neuza, 2014). 


\section{Metodología}

\section{Diseño experimental}

Para la elaboración de una crema exfoliante con el aceite y los residuos sólidos de las semillas de maracuyá se utilizó un análisis de varianza de bloques completos al azar con arreglo factorial de $2 * 3$ y la prueba de significancia Tukey en el programa Infostat. En el factor 1 se consideraron dos concentraciones de aceite de las semillas de maracuyá ( $2 \mathrm{ml}$ y $3 \mathrm{ml}$ ) y, en el factor 2 , tres cantidades de residuo sólido obtenido después de la extracción del aceite de las semillas de maracuyá (1.5; 2.5; $4.0 \mathrm{~g})$.

Tabla 1. Diseño experimental de la crema exfoliante

\begin{tabular}{|l|l|}
\hline \multicolumn{1}{|c|}{ F de $\mathbf{~}$} & gl \\
\hline Total & 17 \\
\hline F1 & 1 \\
\hline F2 & 2 \\
\hline F1*F2 & 2 \\
\hline Repetición & 2 \\
\hline Error & 10 \\
\hline
\end{tabular}

Se trabajó con 6 tratamientos (tabla 2) y 3 repeticiones, las variables analizadas fueron: la extensibilidad, el pH y la estabilidad de la crema exfoliante.

Tabla 2. Tratamientos de la crema exfoliante

\begin{tabular}{|l|l|l|}
\hline \multicolumn{1}{|c|}{ Tratamientos F1 ${ }^{*}$ F2 } & \multicolumn{1}{|c|}{ Aceite F1 $(\mathrm{ml})$} & \multicolumn{1}{c|}{ Residuo sólido F2 (g) } \\
\hline T1 & 2 & 1.5 \\
\hline T2 & 2 & 2.5 \\
\hline T3 & 2 & 4.0 \\
\hline T4 & 3 & 1.5 \\
\hline T5 & 3 & 2.5 \\
\hline T6 & 3 & 4.0 \\
\hline
\end{tabular}

F factores en estudio

\section{Materiales y métodos}

Extracción de aceite de las semillas de maracuyá

El aceite fue extraído por el método químico de solventes en el equipo Soxhlet y por el método mecánico mediante prensado, en una prensa de tornillo sin fin. 


\section{Extracción por prensado}

Para la extracción del aceite de las semillas de maracuyá (Passiflora edulis f flavicarpa Deg) fue necesario eliminar los restos de pulpa adheridos en las semillas y realizar un secado en estufa a $40{ }^{\circ} \mathrm{C}$ por 5 horas, posteriormente se colocaron las semillas en una prensa de tornillo sin fin, de marca Household modelo DSZYJ-200 de fabricación alemana, en la cual se ejecuta un sistema de prensado y compresión, de esta manera, se produce la separación del aceite y del residuo sólido.

El rendimiento en la producción del aceite por este método fue de $22.58 \%$, este rendimiento está dentro de los límites que reportan diferentes estudios sobre el alto porcentaje de aceite vegetal que tienen las semillas de maracuyá, que puede oscilar entre 16.7 \% a 33.5 $\%$, dependiendo de la técnica de extracción y de las zonas climáticas donde fueron cultivadas (Malacrida y Neuza, 2012; Oliveira et al., 2016; Cerón, Osorio y Hurtado, 2012).

El aceite se almacenó a temperatura ambiente en un recipiente de vidrio ámbar, el residuo sólido fue triturado y tamizado hasta alcanzar un diámetro de partícula de $300 \mu \mathrm{m}$, este tamaño de partícula también se consideró en el estudio realizado de una crema exfoliante con moringa (Gutiérrez, Marzoa, Sánchez, Scull, García y Guadarrama, 2016). Además, se debe considerar que estas partículas deben ser esféricas y sin aristas para no causar irritación en la zona de contacto con la piel (Azcoma, 2006) Estos residuos sólidos fueron adicionados a la crema base para producir el efecto exfoliante (W. Li, 2013).

\section{Extracción por el método de Soxhlet}

Para realizar la extracción por el método de Soxhlet fue necesario triturar las semillas hasta un diámetro aproximado de $0.3 \mathrm{~cm}$, para aumentar la superficie de contacto con el solvente; en este se utilizó éter etílico al $99 \%$, de punto de ebullición entre $40{ }^{\circ} \mathrm{C}$ hasta $60^{\circ} \mathrm{C}$. El aceite extraído por este método puede contener residuos del solvente, por lo que fue necesario realizar una destilación por 30 min a $60{ }^{\circ} \mathrm{C}$.

El rendimiento fue del $24.23 \%$, un poco mayor al que se obtiene por el método de prensado, este rendimiento es similar a los encontrados en estudios afines realizados con solventes verdes (23.8 \%) por Cardoso, Davantel y Gimenes (2013).

Sin embargo, el aceite y el residuo sólido obtenidos por este método no fueron utilizados para la preparación de la crema exfoliante, debido a la residualidad de éter a pesar de haber realizado la destilación.

\section{Formulación de la crema exfoliante}

Para la formulación de la crema exfoliante, en primer lugar, se preparó una crema base a la que se añadió el aceite de maracuyá en dos concentraciones y los residuos sólidos obtenidos en la prensa sin fin, en tres cantidades diferentes. Los residuos sólidos son los que caracterizan a la crema exfoliante y aseguran que después de su uso, la piel tenga un aspecto luminoso y más suave al tacto (Azcoma, 2006; Garrote y Bonet, 2008)

La elaboración de la crema base se realizó con los reactivos descritos en la tabla 2. 
Tabla 3. Reactivos para la elaboración de crema exfoliante

\begin{tabular}{|l|l|}
\hline \multicolumn{1}{|c|}{ Reactivos } & \multicolumn{1}{c|}{$\%$} \\
\hline Alcohol cetílico (s) & 4.71 \\
\hline Alcohol estearílico(s) & 2.67 \\
\hline Texapon (gel) & 0.41 \\
\hline Agua destilada (l) & 90.06 \\
\hline Metil parabeno (s) & 0.08 \\
\hline Propil parabeno (s) & 0.005 \\
\hline Propilenglicol (l) & 0.68 \\
\hline Glicerina (l) & 1.39 \\
\hline
\end{tabular}

Se elaboró la crema base siguiendo el procedimiento descrito en el flujo de proceso de la figura 1.

Figura 1. Flujo de proceso de la elaboración de la crema exfoliante

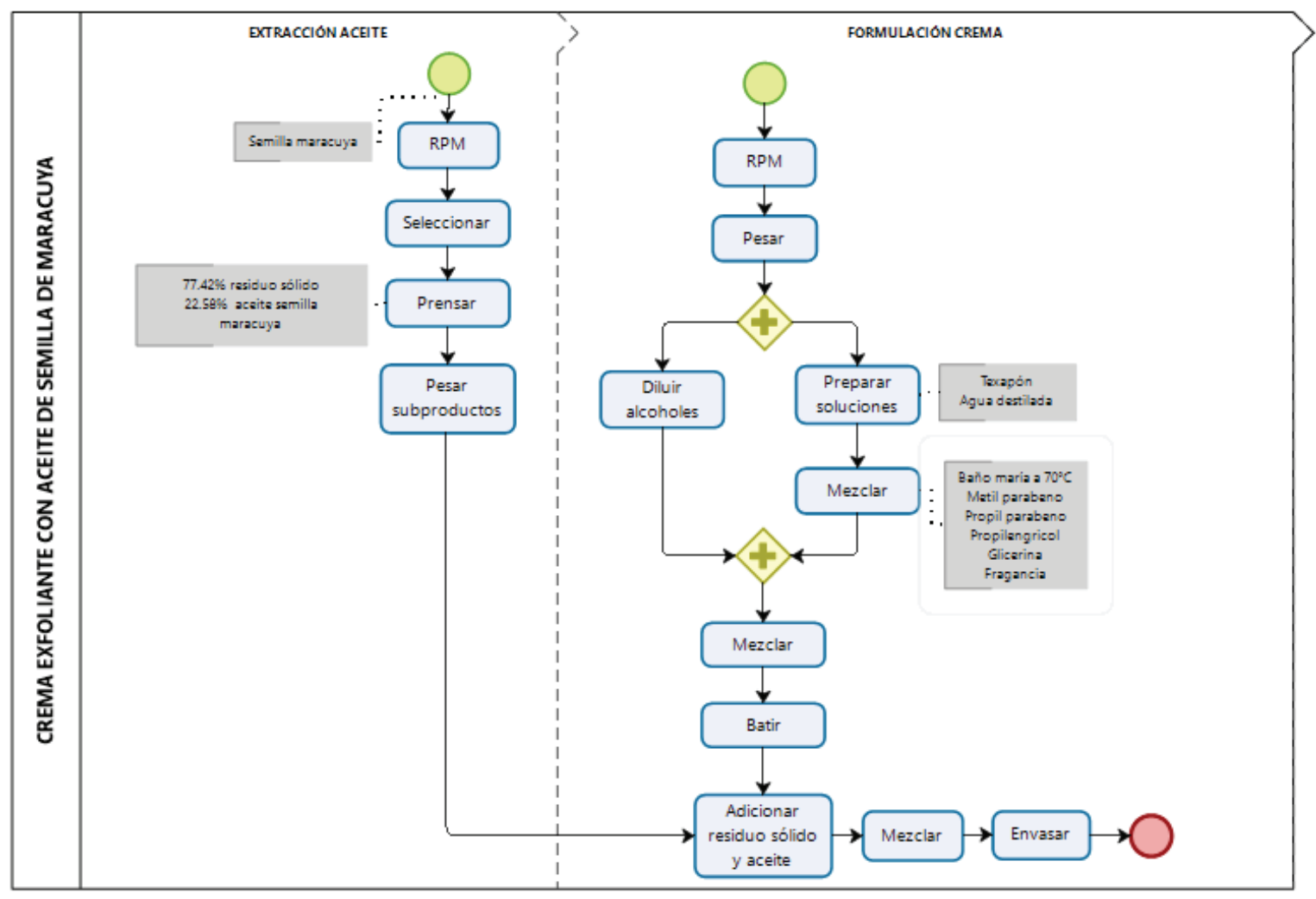

Se preparó $600 \mathrm{~g}$ de crema base, se dividió en muestras de $100 \mathrm{~g}$ a las cuales se agregó los tratamientos de la tabla 1 y, posteriormente, se determinaron las propiedades físico-químicas de las cremas exfoliantes resultantes, según los requerimientos de ANVISA (2005). 


\section{Determinación de las propiedades físico-químicas de la crema exfoliante}

\section{Determinación de la extensibilidad}

Para determinar la extensibilidad en la crema exfoliante, se colocó sobre una hoja de papel milimetrado un porta objetos, para encontrar su centro, en este lugar se colocó $0.25 \mathrm{~g}$ de la crema exfoliante, y se procedió a tapar con un cubre objetos y sobre este se... colocó un peso de $5 \mathrm{~g}$; se esperó por 1 minuto y se midió el radio alcanzado por la crema; se deben tomar varios radios y luego sacar un promedio.

Para calcular el área de extensibilidad se aplicó la siguiente fórmula:

Área de extensibilidad $\left(\mathrm{cm}^{2}\right)=\varpi$ (radio promedio $)^{2}$

(Pérez y Perdomo, 2011; Yambay, 2013).

\section{Determinación del pH}

El pH en la crema exfoliante se midió con el potenciómetro marca Fisher Scientific modelo Ap71; que fue previamente calibrado con soluciones Buffer con $\mathrm{pH} 4$ y pH 7. Estas medidas se tomaron a $20 \mathrm{~g}$ de crema exfoliante de cada uno de los tratamientos manteniendo la temperatura constante de $25^{\circ} \mathrm{C}$. Las mediciones fueron realizadas por triplicado (Anvisa, 2005).

\section{Determinación de la estabilidad}

Para determinar la estabilidad de la crema exfoliante se midió la capacidad de emulsión al someter a $10 \mathrm{~g}$ de la crema de cada uno de los tratamientos a una temperatura de $45^{\circ} \mathrm{C}$ por 45 minutos y colocar en una centrífuga marca Thermocientific Sorvall ST-8 por 30 minutos a 1008 g. Se sacó las muestras de la centrífuga y se observó si existían cambios como la separación de fases o la formación de precipitados (Ramos, 2019; Anvisa, 2005).

\section{Determinación de las características físicas de la crema exfoliante}

Dentro de las características físicas se consideró el color, el olor, el brillo, la arenosidad y ausencia de grumos, estas características macroscópicas son indicadores de una adecuada distribución del tamaño de partícula y de una textura uniforme (USP 36- NF 31, 2013).

El aspecto de la crema exfoliante fue homogéneo y brillante con puntos negros, de color blanco con manchas oscuras y con olor característico a maracuyá, no presentó grumos. Estas características reflejan la correcta interacción entre el aceite y el residuo sólido de las semillas de maracuyá con la crema base, a pesar de que fueron sometidas a centrifugación y altas temperaturas, se mantuvo la estabilidad en todas las formulaciones (Pérez, Nieto, Bilbao, López, González, 2013).

\section{Resultados y discusión}

\section{Pruebas físico químicas}

Para caracterizar a la crema cosmética se evaluaron tres parámetros: las pruebas de extensibilidad, el pH, y la estabilidad de la crema (ANVISA 2005; USP 36- NF 31, 2013). 


\section{Prueba de extensibilidad}

Al analizar los datos de la prueba de extensibilidad mediante un análisis de varianza de bloques completos al azar (DBCA) con arreglo factorial de $2 * 3$ y la prueba de Tukey, se determinó que no existen diferencias significativas entre los tratamientos (tabla 4). Con estos resultados se evidencia que el aceite y los residuos sólidos de las semillas de maracuyá no afectan la extensibilidad de la crema.

Tabla 4. Análisis de varianza en arreglo factorial $2 * 3$

\begin{tabular}{|l|l|l|l|l|l|}
\hline \multicolumn{1}{|c|}{ Fuente } & \multicolumn{1}{c|}{ GL } & \multicolumn{1}{c|}{ SC Ajust. } & \multicolumn{1}{c|}{ MC Ajust. } & \multicolumn{1}{c|}{ Valor F } & \multicolumn{1}{c|}{ Valor $\mathbf{p}$} \\
\hline Aceite de maracuyá & 1 & 0.252 & 0.252 & 0.27 & 0.615 \\
\hline Residuos sólidos & 2 & 3.6374 & 1.8187 & 1.92 & 0.189 \\
\hline $\begin{array}{l}\text { Aceite de maracuyá } \\
\text { Residuos sólidos }\end{array}$ & 2 & 0.4769 & 0.2385 & 0.25 & 0.781 \\
\hline Error & 12 & 11.3672 & 0.9473 & & \\
\hline Total & 17 & 15.7336 & & & \\
\hline
\end{tabular}

Los promedios y la desviación estándar del área de extensibilidad de la crema (tabla 5) reflejan que el tratamiento 4 es el que dio mejores resultados; el tratamiento 6 obtuvo el menor valor en la variable, esto puede suceder porque la cantidad de aceite y residuo sólido es mayor que en los otros tratamientos e incide en la mayor consistencia presentada en la crema exfoliante.

Según USP 36-NF31 (2013) el rango del área de extensibilidad debe estar entre 4.1 y 5 $\mathrm{cm}^{2}$, los resultados de los tratamientos T3, T6, T2 y T5 se encontraron dentro de este rango; el tratamiento T1 y T4 reflejan valores mayores. Todos los tratamientos aseguran que la crema tiene una adecuada distribución en la piel, y no presenta grumos, el aceite de maracuyá y los residuos sólidos no incidieron significativamente sobre la extensibilidad de la crema exfoliante (Fernández-Montes, 2003; Almirall, Fernández, González, 2005).

Estos resultados coinciden con las investigaciones de Yambay (2013) quien determinó la extensibilidad de una crema cicatrizante con berro (Nasturtium officinale) y llanten (Plantago major).

La diferencia de los valores de extensibilidad del presente estudio, con relación a otros estudios, se debe al tamaño de los cristales y a los pesos utilizados en la determinación del área de extensibilidad (Signorelli y Isla, 2005).

Tabla 5. Promedio y desviación estándar para el área de extensibilidad

\begin{tabular}{|l|l|}
\hline \multicolumn{1}{|c|}{ Tratamiento } & \multicolumn{1}{|c|}{$\mathrm{X} \pm \mathrm{D}\left(\mathrm{cm}^{2}\right)$} \\
\hline T6 & $4.64 \pm 0.54 \mathrm{a}$ \\
\hline T3 & $4.65 \pm 0.6 \mathrm{a}$ \\
\hline T2 & $4.93 \pm 1.43 \mathrm{a}$ \\
\hline T5 & $4.96 \pm 0.36 \mathrm{a}$ \\
\hline T1 & $5.37 \pm 1.25 \mathrm{a}$ \\
\hline T4 & $6.06 \pm 1.15 \mathrm{a}$ \\
\hline
\end{tabular}

$\mathrm{X}=$ promedio; $\mathrm{D}=$ desviación estándar 


\section{Potencial hidrógeno}

Con los datos obtenidos de pH se realizó un análisis de varianza con arreglo factorial y se utilizó la prueba de Tukey para determinar las diferencias estadísticas entre los tratamientos. Esto reveló que sí se presentaron diferencias significativas entre los tratamientos: El residuo sólido y las interacciones (tratamientos) influye en el pH de la crema exfoliante (tabla 6), los tratamientos T3, T5 y T6 no presentan diferencias significativas entre ellos (tabla 7) cuyo pH se encontró entre 5.61 y 5.68; los tratamientos $T 5$ y T2 tampoco presentan diferencias significativas entre ellos (5.72 y 5.75), sin embargo, los cinco tratamientos presentan diferencias significativas con el tratamiento T1 que tiene mayor pH (5.87). (Pérez-Bueno, Rodríguez-Perdomo, Morales-Lacarrere, Soler-Roger y de la Paz Martín-Viaña, 2011).

Tabla 6. Análisis de varianza de pH en crema exfoliante

\begin{tabular}{|l|l|l|l|l|l|}
\hline \multicolumn{1}{|c|}{ Fuente de variación } & \multicolumn{1}{c|}{ GL } & \multicolumn{1}{c|}{ SC Ajust. } & \multicolumn{1}{c|}{ MC Ajust. } & \multicolumn{1}{c|}{ Valor $\mathbf{F}$} & \multicolumn{1}{c|}{ Valor $\mathbf{p}$} \\
\hline Aceite de maracuyá & 1 & 0.003756 & 0.003756 & 0.79 & 0.393 \\
\hline Reciduos sólidos & 2 & 0.087678 & 0.043839 & 9.16 & 0.004 \\
\hline $\begin{array}{l}\text { Aceite de maracuyá * } \\
\text { Reciduos sólidos }\end{array}$ & 2 & 0.029144 & 0.014572 & 3.05 & 0.045 \\
\hline Repetición & 2 & 0.029144 & 0.014572 & 3.05 & 0.085 \\
\hline Error & 10 & 0.0574 & 0.004783 & & \\
\hline Total & 17 & 0.177978 & & & \\
\hline
\end{tabular}

El tratamiento T3 es el que presenta menor valor de $\mathrm{pH}$ y el T1, por el contrario, reporta el pH más alto, estos valores se encuentran en un rango entre 5.61 hasta 5.87. La diferencia entre estos dos tratamientos no es la cantidad de aceite de semilla de maracuyá sino la cantidad de residuo sólido. Sin embargo, estos valores de pH están dentro de los valores de acidez de la superficie de la piel que oscila entre 4 y 6 (Schmid-Wendtner, Korting, 2006).

Según USP 36-NF31 (2013), el valor de pH de la crema cosmética debe encontrarse entre 5.5 y 7.0, todos los tratamientos se encuentran dentro de este rango. Estos resultados coinciden con los reportados para una crema exfoliante a base de moringa, cuyos resultados de $\mathrm{pH}$ se encontraron entre 5.71 y 5.76 (Gutiérrez, Marzoa, Sánchez, Scull, García, Guadarrama, 2016; Soler, Rodríguez, Pérez, Sánchez, Lazo, 2007).

Los valores de $\mathrm{pH}$ en los diferentes tratamientos, se pueden ver influenciados por el pH del aceite de las semillas de maracuyá que es de 4.05 y por la presencia de residuos sólidos (Pantoja-Chamorro, Hurtado-Benavides y Martínez-Correa, 2017).

Tabla 7. Prueba de Tukey de pH de crema exfoliante

\begin{tabular}{|l|l|}
\hline \multicolumn{1}{|c|}{ Tratamiento } & \multicolumn{1}{|c|}{$\mathbf{X} \pm \mathbf{D}$} \\
\hline T3 & $5.61 \pm 0.05 \mathrm{a}$ \\
\hline T6 & $5.68 \pm 0.02 \mathrm{a}$ \\
\hline T5 & $5.68 \pm 0.01 \mathrm{a}$ \\
\hline T2 & $5.72 \pm 0.04 \mathrm{ab}$ \\
\hline T4 & $5.75 \pm 0.00 \mathrm{ab}$ \\
\hline T1 & $5.87 \pm 0.16 \mathrm{~b}$ \\
\hline
\end{tabular}

$\mathrm{X}=$ promedio; $\mathrm{D}=$ desviación estándar 
Según la norma RTE INEN 2867 (2015), el pH para cremas ácidas debe ser menor a 3 y para cremas alcalinas mayor a 10, la crema exfoliante con aceite y residuo sólido de las semillas de maracuyá, por tener un pH entre 5.61 y 5.87 se considera una crema ligeramente ácida, por tanto, se puede aplicar como exfoliante de rostro y cuerpo (Ferrari, Colussi y Ayub, 2004).

El pH de 5.61 a 5,87 de la crema exfoliante favorece la estabilidad de los compuestos bioactivos presentes en las semillas de maracuyá, estos poseen características antioxidantes que dan un valor agregado a la crema exfoliante (Malacrida y Jorge, 2012).

\section{Estabilidad}

Después de sometida la crema exfoliante a la centrifugación, se la evaluó visualmente, se determinó que no presentó cambios significativos en el cremado o coalescencia, no se presentaron precipitaciones, separación de fases ni formación de escamas en ninguno de los tratamientos, lo que comprueba la estabilidad de las formulaciones (Heberle, Dos Santos y M Magri, 2012). Además, en el estudio de Gutiérrez, Marzoa, Sánchez, Scull, García y Guadarrama (2016) también se demostró que las cremas exfoliantes son estables.

Existió una elevada compatibilidad entre la formulación de la crema, el aceite de maracuyá y el residuo sólido, la temperatura y la centrifugación a la que fue sometida la crema exfoliante no provocó movimiento de partículas internas ni estrés que provoquen inestabilidades (Ramos, Acosta, Reboredo, Torres y Cabrer, 2013).

La crema exfoliante de aceite y el residuo sólido de las semillas de maracuyá no alteraron la capacidad de emulsión de la crema exfoliante, por lo tanto, se considera de característica estable (Cruz y Meléndez Zepeda, 2004) y cumple con lo establecido por la normativa ANVISA (2005) que menciona que las cremas cosméticas deben ser productos estables.

\section{Conclusiones}

La aplicación de los semielaborados de semillas de maracuyá, aceite y residuo sólido obtenidos de la extracción, en una crema exfoliante, permiten aprovechar los residuos agroindustriales de la elaboración de productos vegetales para el consumo humano.

Las semillas de maracuyá presentan en su composición un elevado contenido de proteína (12.23 \%) y aceites poliinsaturados (33 \%) lo que confiere a las mismas, características de agente acondicionador de la piel y el cabello.

La crema exfoliante a base de aceite y residuos sólidos de las semillas de maracuyá, presentó características de $\mathrm{pH}$, extensibilidad y estabilidad acordes a la normativa de productos cosméticos NTE-INEN 2867 y USP 36-NF31.

El pH de la crema exfoliante se mantuvo entre 5.61 y 5.87. Estos valores se encuentran dentro del rango normado para cremas cosméticas, 4.5 a 7 (USP 36-NF31): por lo tanto, el producto se puede utilizar tanto para el rostro como para el cuerpo.

La estabilidad de la crema exfoliante con aceite y residuos de semillas de maracuyá se mantuvo en todos los tratamientos, no se presentó separación de fases ni precipitaciones 0 escamas. La presencia del aceite y los residuos de las semillas de maracuyá no afectó la estabilidad de la emulsión.

La distribución de la crema exfoliante sobre la piel, se comprobó con la prueba de extensibilidad cuyos resultados superaron los $4 \mathrm{~cm}^{2}$. Se comprobó que el aceite y el residuo sólido de las semillas de maracuyá no afectan la extensibilidad de la crema. 
El tratamiento 4 ( $3 \mathrm{ml}$ de aceite y $1.5 \mathrm{~g}$ de residuo sólido de la semilla de maracuyá) presentó mejores resultados en las pruebas aplicadas. Las pruebas de extensibilidad, estabilidad y $\mathrm{pH}$ de la crema exfoliante, se encontraron dentro de los límites aceptables

\section{Agradecimientos}

Los autores agradecen al doctor Trevor Jackson investigador de AgResearch por el aporte técnico, y al Gobierno de Nueva Zelandia (MFAT New Zealand) por el financiamiento. También agradecemos a los organizadores del II Simposio Internacional de Producción Integrada de Frutas 2019, por permitirnos difundir los resultados de este estudio

\section{Bibliografía}

Agencia Nacional de Vigilancia Sanitaria (2005) Guía de estabilidad de productos cosméticos. Recuperado de:

Portal.anvisa.gov.br/documents/33880/4967127/Biblioteca+dos+Temas+de+Cosméticos_Versão2018_ Portal.pdf/75acd4c5-2926-4338-a692-8906cb76cd16

Almirall, I., Fernández, T., González, H. M. (2005). Diseño de una crema para masajes con extracto de spirulina cubana. Rev. Cubana Farm, 39 (3):15-18.

Azcona, L. (2006) Dermofarmacia. Exfoliación. Farmacia profesional. Elsevier, 20 (7):56-59.

Cardoso, R., Davantel, S. y Gimenes, M. (2013). The extraction of passion fruit oil with green solvents. J. Food Eng, 117 (4): 458-463.

Cerón, A., Osorio, O., y Hurtado, A. (2012). Identificación de ácidos grasos contenidos en los aceites en los aceites a partir de semillas de tres diferentes aceites de frutas. Acta Agron, 61 (2): 126-132.

Cruz, R. L., y Meléndez Zepeda, C. L. (2004). Obtención, refinación y caracterización del aceite de la semilla de Passiflora edulis flavicarpa (maracuyá) (Doctoral dissertation, Universidad de El Salvador).

Da Silva, A., \& Neuza, J. (2014). Bioactive compounds of the lipid fractions of agro-industrial waste. Food Research International, 66: 493-500.

García, M. (2002). Guía técnica. Cultivo de maracuyá amarillo. La libertad, San Salvador:. Centa.

Garrote, A. y Bonet, R. (2008). Exfoliantes de nueva generación. Propuestas innovadoras. O FFA R M, 27 (9): 55-60.

Gutiérrez, Y. I., Marzoa, N., Sánchez, A., Scull, R., García, G. y Guadarrama, D. (2016). Propuesta de una crema exfoliante a partir de la torta de las semillas de Moringa oleifera (Lam.). Proposalof anexfoliant cream from cake seeds of Moringa oleifera (Lam.). Revista de Ciencias Farmacéuticas y Alimentarias, 2 (2): 1-13.

Heberle, G., Dos Santos, M. A. y Magri, S. (2012). Cosmetic formulations containing blueberry extracts (Vaccinium myrtillus l.). tojsat, 2 (1): 1-6.

Ferrari, R. A., Colussi F, Ayub RA. (2004). Caracterización de subproductos de la industrialización de maracuyá utilización de semillas. Rev Bras Frutic. 26 (1): 101-102.

Fernández-Montes, E. A. (2003). Control de calidad de fórmulas dermatológicas. Farmacia Profesional, 17(2), 70-74.

Leao, K., Sampayo, K., Pagani, A., \& Da Silva, M. (2014). Odor potency, aroma profile and volatiles composition of cold pressedoil from industrial passion fruit. Ind Crop Prod, 280-286.

Lillini, G., Pasquali, R., Pedemonte, C., Bregni, C., \& Lavaselli, S. (2016). Estudio de la estabilidad de emulsiones con estructuras líquido-cristalinas, y su aplicación farmacéutica mediante el agregado de un principio activo liposoluble: Econazol. Revista Colombiana de Ciencias Químico - Farmacéuticas, 95. 
Liu, G., Xu, X., Gong, Y., He, L., \& Gao, Y. (2012). Effects of supercrítical CO2 extraction parameters on chemical composition and free radical-scavenging activity of pomegranate (Punica granatum L.) seed oil. Food Bioprod Process, 573-578.

Malacrida, C., \& Neuza, J. (2012). Yellow passion fruit seed oil (Passiflora edulis F. flavicarpa): Physical and chemical characteristics. Braz Arch Biol, 127-134.

Oliveira, D., Angonesea, M., Gómez, C., \& Ferreira, S. (2016). Valorization of passion fruit (Passiflora edulis sp.) by-products: Sustainable recovery and biologycal activities. J Supercrit Fluid, 55-62.

Pantoja, A., Hurtado, A., y Martínez, H. (2017). Caracterización de aceite de semillas de maracuyá (Passiflora edulis Sims.). Acta Agronómica, 66 (2): 178-185.

Pérez, T., \& Perdomo, Y. (2011). Comportamiento reológico y extensibilidad de una formulación semisólida a partir del extracto acuoso de Rhizophora mangle L. Tecnol. Ciencia Ed. (IMIQ), 75-79.

Pérez PR, Nieto OMA, Bilbao OR, López AT, González LC. (2013). Diseño de una crema regeneradora con quitina para después del bronceado. Revista Cubana de Farmacia 47 (2): 239-251.

Pérez-Bueno, T., Rodríguez-Perdomo, Y., Morales-Lacarrere, I., Soler-Roger, D. M. y de la Paz Martín-Viaña, N. (2011). Comportamiento reológico y extensibilidad de una formulación semisólida a partir del extracto acuoso de Rhizophora mangle L. Tecnología, Ciencia, Educación, 26 (2): 75-79.

Ramos, P. P., Acosta, O. M. N., Reboredo, O. B., Torres, A. L. y Cabrer, L. G. (2013). Diseño de una crema regeneradora con quitina para después del bronceado. Revista Cubana de Farmacia, 47 (2): 239-251.

Ramos, P. P. (2019). S3-Ciencias Cosméticas/Cosmetic Sciences. Revista de Ciencias Farmacéuticas y Alimentarias, 5 (1): 6.

Servicio Ecuatoriano de Normalización. (2015). Resolución 15057 NTE INEN 2867. Productos cosméticos requisitos. Quito.

Schmid-Wendtner MH, Korting HC. (2006) The pH of the skin surface and its impact on the barrier function. Pharmacol Appl skin physiol, 19: 296-302.

Signorelli, I., y Isla, M. (2005). Elaboración de una crema para uso tópico a base de Urtica dioica L. Revista de la Facultad de Farmacia, 47 (2): 26-32.

Soler, D.M., Rodríguez, Y., Pérez, T., Sánchez, L. y Lazo, R. (2007). Extensibilidad en la preformulación de un gel a partir de Rhizophora mangle L. En Memorias del III Simposio Internacional de Química. CDROM ISBN 978-959-250-337-3. Sociedad Cubana de Química. La Habana, Cuba.

United States Pharmacopeial (2013). Vol 1, USP 36: NF 31United States Pharmacopeia. The National Formulary. Rockville, Maryland: United States Pharmacopeia Convention Inc.

W. Li, Zhai, Z. Pang, Q. Kong, L. y Zhou A. . (2013). Influence of exfoliating facial cleanser on the bio-tribiological properties of human skin. WEAR, 301(1-2): 353-361.

Yambay Calderón, P. F. (2013). Elaboración y control de calidad de una crema a base de los extractos hidroalcohólicos de berro (Nasturtium officinale) y llántén (Plantago major) y comprobación de su actividad cicatrizante en heridas inducidas en ratones (Bachelor's thesis).

Zeraik, M., Pereira, C., Zuin, V. y Yariwake, J. (2010). Maracujá: un alimento funcional? Rev Bras Farmacogn, 429-471 